\title{
DESINGULARISATION OF ORBIFOLDS OBTAINED FROM SYMPLECTIC REDUCTION AT GENERIC COADJOINT ORBITS
}

\author{
KLAUS NIEDERKRÜGER AND FEDERICA PASQUOTTO
}

\begin{abstract}
We show how to construct a resolution of symplectic orbifolds obtained as quotients of presymplectic manifolds with a torus action. As a corollary, this allows us to desingularise generic symplectic quotients. Given a manifold with a Hamiltonian action of a compact Lie group, symplectic reduction at a coadjoint orbit which is transverse to the moment map produces a symplectic orbifold. If moreover the points of this coadjoint orbit are regular elements of the Lie coalgebra, that is, their stabiliser is a maximal torus, the result for torus quotients may be applied to obtain a desingularisation of these symplectic orbifolds. Regular elements of the Lie coalgebra are generic in the sense that the singular strata have codimension at least three.

Additionally, we show that even though the result of a symplectic cut is an orbifold, it can be modified in an arbitrarily small neighbourhood of the cut hypersurface to obtain a smooth symplectic manifold.
\end{abstract}

\section{INTRODUCTION}

Let $G$ be a compact Lie group, and let $(W, \omega)$ be a Hamiltonian $G$-manifold with moment map $\mu: W \rightarrow \mathfrak{g}^{*}$. If $\mu$ is transverse to the coadjoint orbit $\operatorname{Ad}(G)^{*} \nu$, then the preimage $\mathcal{O}\left(\operatorname{Ad}(G)^{*} \nu\right):=$ $\mu^{-1}\left(\operatorname{Ad}(G)^{*} \nu\right)$ of the coadjoint orbit is a smooth $G$-invariant submanifold. It also follows that every element on the coadjoint orbit $\operatorname{Ad}(G)^{*} \nu$ is a regular value of the moment map $\mu$, and so the $G$-action is locally free, because for $X \in \mathfrak{g}$ the infinitesimal generator $X_{W}$ satisfies the equation $i_{X_{W}} \omega=\langle d \mu \mid X\rangle$, so that $X_{W}$ cannot vanish at regular points of the moment map $\mu$. The quotient

$$
\mathcal{O}\left(\operatorname{Ad}(G)^{*} \nu\right) / / G:=\mu^{-1}\left(\operatorname{Ad}(G)^{*} \nu\right) / G
$$

is an orbifold. The easiest way to define the canonical symplectic structure on $\mathcal{O}\left(\operatorname{Ad}(G)^{*} \nu\right) / / G$ is by using the isomorphism

$$
\mathcal{O}\left(\operatorname{Ad}(G)^{*} \nu\right) / / G \cong \mu^{-1}(\nu) / \operatorname{Stab}(\nu) .
$$

The 2-form $\omega$ descends to a non-degenerate form on the quotient on the right hand side, because the tangent space to the $\operatorname{Stab}(\nu)$-orbits spans the kernel of $\left.\omega\right|_{T \mu^{-1}(\nu)}$.

In this paper we prove the following result, which is a generalisation of the result in [NP07.

Theorem 1. Let $(M, \omega)$ be a symplectic orbifold arising as the quotient of a presymplectic $\mathbb{T}^{k}$ manifold by the given torus action and let $X$ be the set of orbifold singularities of $M$. Assume that $X$ is compact: then for any neighbourhood $U$ of $X$ there exists a symplectic resolution of $M$ supported on $U$.

Definition. An element $\nu$ in the Lie coalgebra $\mathfrak{g}^{*}$ is called regular if its stabiliser with respect to the coadjoint action is a maximal torus.

In fact most elements of the Lie coalgebra are regular: their complement consists of the union of finitely many subspaces of $\mathfrak{g}^{*}$, the singular strata, which have codimension at least 3 . Using the isomorphism (1.1) we can apply the theorem to a symplectic reduction at a generic element $\nu \in \mathfrak{g}^{*}$ to obtain the following

Corollary 2. Let $G$ be a connected compact Lie group, and let $(W, \omega)$ be a Hamiltonian $G$ manifold with moment map $\mu$. Choose an element $\nu \in \mathfrak{g}^{*}$ that is a regular value of $\mu$ and a regular element of the Lie coalgebra. The reduced space $M:=\mathcal{O}\left(\operatorname{Ad}(G)^{*} \nu\right) / / G$ is a symplectic orbifold and, provided that the set $X$ of orbifold singularities is compact, it admits a symplectic resolution supported on an arbitrarily small neighbourhood of $X$. 
Acknowledgements. K. Niederkrüger is working at the ENS de Lyon, where he is being funded by the Agence Nationale de la Recherche (ANR) project Symplexe. F. Pasquotto is working at the Vrije Universiteit Amsterdam and is supported by VENI grant 639.031 .620 of the Nederlandse Organisatie voor Wetenschappelijk Onderzoek (NWO).

We would like to thank the Centre International de Rencontres Mathématiques at Luminy for their hospitality in the summer of 2008, while we were working on the main ideas for this paper. We are also grateful to Eugene Lerman for his patience in answering our questions and for asking in turn more questions that stimulated us to think further.

\section{Presymplectic manifolds and symplectic orbifolds}

In this section, $G$ will denote a compact connected Lie group and $\mathfrak{g}$ its Lie algebra. If $G$ acts on a manifold $P$, then there exists a homomorphism

$$
\mathfrak{g} \rightarrow \mathfrak{X}(P), \quad X \mapsto X_{P}(p)=\left.\frac{d}{d t}\right|_{t=0} \exp (t X) * p .
$$

The vector $X_{P}$ is called the infinitesimal action of $X \in \mathfrak{g}$.

Definition. Let $P$ be a $(2 n+k)$-dimensional smooth manifold and assume it admits a locally free $G$-action and a closed 2 -form $\omega_{P}$ such that:

(i) $\operatorname{dim} G=k$;

(ii) $\omega_{P}^{n} \neq 0$;

(iii) the infinitesimal action of $\mathfrak{g}$ spans the kernel of $\omega_{P}$.

We call $P$ a presymplectic $G-$ manifold.

Definition. A symplectic orbifold $M$ is a Hausdorff, second countable topological space, equipped with an atlas of uniformizing charts $\left(\widetilde{U}_{i}, \Gamma_{i}, \varphi_{i}, \omega_{i}\right)$, where $\widetilde{U}_{i}$ is an open connected subset of $\mathbb{R}^{2 n}, \Gamma_{i}$ is a finite group of symplectomorphisms of $\left(\widetilde{U}_{i}, \omega_{i}\right)$ and $\varphi_{i}: \widetilde{U}_{i} \rightarrow M$ induces a homeomorphism from $\widetilde{U}_{i} / \Gamma_{i}$ to $U_{i} \subset M$. These charts are required to cover $M$ and to satisfy the following compatibility condition: if $x \in \widetilde{U}_{i}$ and $y \in \widetilde{U}_{j}$ are such that $\varphi_{i}(x)=\varphi_{j}(y)$ then there exists a symplectomorphism from a neighbourhood of $x$ onto a neighbourhood of $y$ whose composition with $\varphi_{j}$ is $\varphi_{i}$.

Definition. Let $M_{1}$ and $M_{2}$ be orbifolds with uniformizing charts $\left\{\left(\widetilde{U}_{i}, \Gamma_{i}, \varphi_{i}\right)\right\}$ and $\left\{\left(\widetilde{V}_{j}, \Lambda_{j}, \psi_{j}\right)\right\}$, respectively. A smooth orbifold map $f: M_{1} \rightarrow M_{2}$ is a continuous map such that if $x \in \widetilde{U}_{i}$ and $y \in \widetilde{V}_{j}$ are such that $f\left(\varphi_{i}(x)\right)=\psi_{j}(y)$, then there exists a smooth map $\widetilde{f}$ from a neighbourhood of $x$ to a neighbourhood of $y$ such that $\psi_{j} \circ \widetilde{f}=f \circ \varphi_{i}$.

A $G$-orbifold $M$ is a smooth orbifold map $G \times M \rightarrow M$ with the usual properties of an action. The definition of presymplectic $G$-orbifold is also analogous to the corresponding one for manifolds.

Lemma 1. Let $P$ be a presymplectic $G_{1} \times G_{2}$-manifold. Then $P / G_{1}$ is a presymplectic $G_{2}-$ orbifold. Moreover, $P /\left(G_{1} \times G_{2}\right)$ and $\left(P / G_{1}\right) / G_{2}$ are isomorphic as symplectic orbifolds.

The proof of the lemma above is analogous to the case of smooth manifolds with free group actions. In our situation, though, one has to use the symplectic orbifold slice theorem, as stated in [T97.

\subsection{Symplectic resolutions.}

Definition. Let $M$ be an orbifold with singular set $X$. A resolution of $M$ consists of a smooth manifold $\widetilde{M}$ and a continuous surjective map $p: \widetilde{M} \rightarrow M$ which is a diffeomorphism on the complement of the singular set.

If $(M, \omega)$ is a symplectic orbifold (presymplectic $G$-orbifold) and $U$ is a neighbourhood of the singular set $X$, then a symplectic (presymplectic) resolution of $M$ supported on $U$ consists of a smooth symplectic (presymplectic $\left.G^{-}\right)$manifold $(\widetilde{M}, \widetilde{\omega})$ and a $(G$-equivariant) resolution $p: \widetilde{M} \rightarrow M$ such that $p^{*} \omega=\widetilde{\omega}$ on the complement of $U$. 


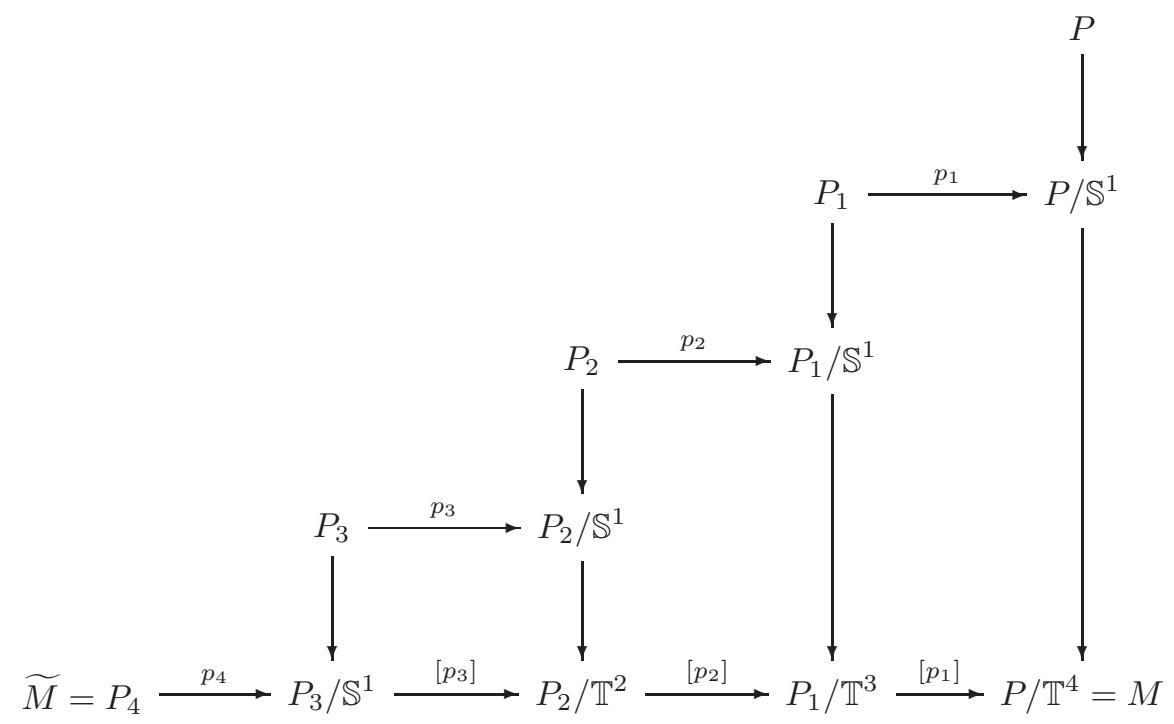

Figure 1. Resolution scheme applied to a presymplectic $\mathbb{T}^{4}$-manifold $(P, \omega)$.

\section{Construction of the Resolution}

3.1. Strategy. Let $M$ be a symplectic orbifold arising as the quotient of a presymplectic $\mathbb{T}^{k}$ manifold $P$ by the given group action, and let $U$ be a neighbourhood of the set of orbifold singularities of $M$. If we split off an $\mathbb{S}^{1}$-factor from the $k$-dimensional torus and view $M$ as the quotient of a presymplectic $\mathbb{S}^{1} \times \mathbb{T}^{k-1}$-manifold, by Lemma 1 we have that $P / \mathbb{S}^{1}$ is a presymplectic $\mathbb{T}^{k-1}$-orbifold and $\left(P / \mathbb{S}^{1}\right) / \mathbb{T}^{k-1} \cong M$. If the $\mathbb{S}^{1}$-action on $P$ happens to be free, then $P / \mathbb{S}^{1}$ is a smooth presymplectic $\mathbb{T}^{k-1}$-manifold and we can split off another $\mathbb{S}^{1}$-factor. If the action is only locally free, in Lemma 2 below we show that one can construct a presymplectic resolution $P_{1}$ of $P / \mathbb{S}^{1}$ (with $\mathbb{T}^{k-1}$-action) supported on an arbitrarily small neighbourhood $U_{1}$ of the set of singular points of $P / \mathbb{S}^{1}$. In particular, we can choose $U_{1}$ in such a way that its image under the $\mathbb{T}^{k-1}$-action is contained in $U$. From this it follows that the map $p_{1}: P_{1} \rightarrow P / \mathbb{S}^{1}$ induces a surjective map $\left[p_{1}\right]: P_{1} / \mathbb{T}^{k-1} \rightarrow\left(P / \mathbb{S}^{1}\right) / \mathbb{T}^{k-1} \cong M$ which is a diffeomorphism outside the set of singular points of $M$ and a symplectomorphism outside $U$.

If we iterate this step (see Fig. (1), we get a sequence of manifolds $P_{1}, \ldots, P_{k}$ such that:

(i) $P_{i}$ is a presymplectic $\mathbb{T}^{k-i}$-manifold;

(ii) $p_{i}: P_{i} \rightarrow P_{i-1} / \mathbb{S}^{1}$ is a presymplectic resolution supported on $U_{i}$ with an action of $\mathbb{T}^{k-i}$ such that $U_{i} / \mathbb{T}^{k-i} \subset\left(\left[p_{1}\right] \circ \cdots \circ\left[p_{i-1}\right]\right)^{-1}(U)$;

(iii) the induced maps $\left[p_{i}\right]: P_{i} / \mathbb{T}^{k-i} \rightarrow P_{i-1} / \mathbb{T}^{k-i+1}$ can be composed to obtain a symplectic resolution $[p]=\left[p_{1}\right] \circ\left[p_{2}\right] \circ \cdots \circ\left[p_{k}\right]: \widetilde{M}=P_{k} \rightarrow M=P / \mathbb{T}^{k}$ of $M$ supported on $U$.

Lemma 2. Let $(P, \omega)$ be a presymplectic $\mathbb{S}^{1} \times G$-manifold. Let $U$ be a neighbourhood of the set of singular points for the $\mathbb{S}^{1}$-action. Then we find a presymplectic $\mathbb{S}^{1} \times G$-manifold $(\widetilde{P}, \widetilde{\omega})$ such that the $\mathbb{S}^{1}$-action is free, and such that there is a map $p: \widetilde{P} / \mathbb{S}^{1} \rightarrow P / \mathbb{S}^{1}$ that is a G-equivariant presymplectic resolution of $P / \mathbb{S}^{1}$ supported on $U$.

The rest of Section 3 will be devoted to the proof of this lemma. For this we have to adapt the symplectic cut [Ler95 to our presymplectic setting. Roughly speaking we construct an auxiliary circle action in a tubular neighbourhood of the stratum of singularities with highest isotropy group. Then we cut out a smaller neighbourhood, and using the auxiliary action we collapse the boundary of the cavity to reobtain a closed manifold. With this construction, we have removed the points with highest isotropy groups, and then we can reapply the same steps to reduce successively the order of the worst singular points, until none are left. 
3.2. Reducing the order of the singularities of the $\mathbb{S}^{1}$-action. Choose an $\mathbb{S}^{1} \times G$-invariant splitting of the tangent bundle,

$$
T P=\operatorname{ker} \omega_{P} \oplus \Omega^{P} .
$$

Since $\Omega^{P}$ is a symplectic subbundle, it admits an $\mathbb{S}^{1} \times G$-invariant almost complex structure $J$ that is compatible with $\left.\omega_{P}\right|_{\Omega^{P}}$ (see for example [MS98, Section 5.5]). Consider the metric on $\Omega^{P}$ given by $g=\omega_{P}(J-,-)$, and extend it to an invariant metric on $T P$ such that $\operatorname{ker} \omega_{P} \perp \Omega^{P}$.

Consider the stratification $\left\{P_{k}\right\}$ of the singular set of the $\mathbb{S}^{1}$-action on $P$, with $P_{k}=\{p \in P \mid$ $\left.\operatorname{Stab}_{\mathbb{S}^{1}}(p) \cong \mathbb{Z}_{k}\right\}$. If $k$ is maximal, that is, $P_{k^{\prime}}=\emptyset$ for $k^{\prime}>k$, then $P_{k}$ is a closed, $\mathbb{S}^{1} \times G$-invariant submanifold of $P$, of codimension at least 2. By restricting to one component, we may further assume $P_{k}$ to be connected. A model of a neighbourhood of $P_{k}$ is given by a neighbourhood of the zero section in the total space of its normal bundle $\nu_{k}$. The linearisation of the $\mathbb{S}^{1} \times G$-action on $P$ defines an action on $\nu_{k}$ that is equivalent to the given one on $P$ (in the sense that the exponential map defines an equivariant diffeomorphism from a neighbourhood of the zero section in $\nu_{k}$ to a neighbourhood of $P_{k}$ in $P$ ). Later it will be necessary to introduce an auxiliary circle action. To avoid confusions, from now on we will call the given $\mathbb{S}^{1}$-action the $\beta$-action and we will write it as $\lambda *_{\beta} v$ for $\lambda \in \mathbb{S}^{1}$ and $v \in \nu_{k}$, whereas we will write $g *_{G} v$ for the $G$-action.

Let $x \in P_{k}$ be a point in the minimal stratum. The $\mathbb{S}^{1}$-stabiliser $\operatorname{Stab}_{\mathbb{S}^{1}}(x) \cong \mathbb{Z}_{k}$ acts by isometric $J$-linear transformations on $\Omega_{x}^{P}$, hence there exists an isomorphism between the Hermitian vector spaces $\left(\Omega_{x}^{P}, J, g\right)$ and the standard Hermitian space $\mathbb{C}^{n}$ such that the linearised $\mathbb{Z}_{k}$-action takes the form

$$
\lambda *_{\beta} \mathbf{z}=\left(\lambda^{\widetilde{a}_{1}} z_{1}, \ldots, \lambda^{\widetilde{a}_{n}} z_{n}\right), \quad \lambda \in \mathbb{Z}_{k}, \mathbf{z} \in \mathbb{C}^{n}, \widetilde{a}_{1}, \ldots, \widetilde{a}_{n} \in \mathbb{Z} .
$$

Without loss of generality we may assume that $0=\widetilde{a}_{1}=\cdots=\widetilde{a}_{m}<\widetilde{a}_{m+1} \leq \cdots \leq \widetilde{a}_{n}<k$ for some $m$. The first $m$ directions span the space $\Omega_{x}^{P} \cap T_{x} P_{k}$, and since the others are orthogonal, they coincide with the fibre $\nu_{k}(x)$. In particular, it follows that $\nu_{k}$ is a $J$-complex bundle with a fibrewise $J$-linear $\mathbb{Z}_{k}$-action that commutes with the $\mathbb{S}^{1} \times G$-action.

Denote by $a_{1}<\cdots<a_{l}$ the distinct exponents occurring in the normal form (3.1) for the action: $\nu_{k}$ splits thus into a direct sum of $\mathbb{S}^{1} \times G$-invariant subbundles

$$
\nu_{k}=E_{1} \oplus \cdots \oplus E_{l},
$$

where $E_{i}(x)$ denotes the eigenspace corresponding to the eigenvalue $\lambda^{a_{i}}$ in the fibre at the point $x$. The splitting is well defined for each component of $P_{k}$. This allows us to extend the $\mathbb{Z}_{k}$-action to an auxiliary circle action $\varphi$ by setting for any $\lambda \in \mathbb{S}^{1}$

$$
\lambda *_{\varphi} v:=\lambda^{a_{1}} v_{1}+\cdots+\lambda^{a_{l}} v_{l},
$$

where $v=v_{1}+\cdots+v_{l}$ is a splitting with respect to the eigenspaces defined above. This $\mathbb{S}^{1}$-action is fibrewise and $J$-linear, and commutes with the original $\mathbb{S}^{1} \times G$-action. The presymplectic form $\omega_{P}$ is $\varphi$-invariant at points of $P_{k}$, but unfortunately it does not need to be invariant at other points. By averaging $\omega_{P}$ over the $\varphi$-action, we obtain a closed 2-form $\omega$ which is invariant with respect to both the $\mathbb{S}^{1} \times G$ - and the $\varphi$-action, and such that the $\mathbb{S}^{1} \times G$-orbits still lie in the kernel of $\omega$. At points of $P_{k}$, where $\omega_{P}$ was already $\varphi$-invariant, we did not change it by averaging, and so we have $\omega^{n} \neq 0$. It follows that there is a small neighbourhood of the zero section, where $\omega$ will be $\mathbb{S}^{1} \times G$-presymplectic.

Proposition 3. There exist neighbourhoods $U_{1}, U_{2}$ of $P_{k}$ in $\nu_{k}$ and an $\mathbb{S}^{1} \times G$-equivariant diffeomorphism $\Psi: U_{1} \rightarrow U_{2}$ such that

$$
\Psi^{*} \omega=\omega_{P}
$$

The proof of this statement is a variation on the proof of the analogous statement in the symplectic case, but for completeness we have included it in the appendix. This proposition shows that we can pull-back the $\varphi$-action to a neighbourhood of $P_{k}$ where it gives us an auxiliary action for which the original presymplectic form $\omega_{P}$ is invariant. Equivalently we can work with the action $\varphi$ we have defined above and the averaged symplectic form $\omega$. In this paper we choose to do the latter. 
Proposition 4. There exists a neighbourhood $U$ of $P_{k}$ in $\nu_{k}$ and a non negative $\mathbb{S}^{1} \times G$-invariant Morse-Bott function $\mu_{\varphi}: U \rightarrow \mathbb{R}$ such that

- $i_{X_{\varphi}} \omega=d \mu_{\varphi}$

- $\mu_{\varphi}$ vanishes only on the zero section of $\nu_{k}$, and it is strictly increasing in radial fibre direction.

Proof. Since $\omega$ is $\varphi$-invariant, one has that $d i_{X_{\varphi}} \omega=\mathcal{L}_{X_{\varphi}} \omega=0$. For the time being, let $U$ be any tubular neighbourhood of $P_{k}$, where $\omega$ is defined. The closed 1 -form $i_{X_{\varphi}} \omega$ represents a class in $H^{1}(U)$ which vanishes if we pull it back to the zero section $P_{k}$ : Given that $H^{1}(U) \cong H^{1}\left(P_{k}\right)$, it follows that $i_{X_{\varphi}} \omega$ is exact on $U$, i.e., there exists a function $\mu_{\varphi}$ such that $i_{X_{\varphi}} \omega=d \mu_{\varphi}$. The function $\mu_{\varphi}$ is uniquely defined up to an additive constant (which we may choose such that $\mu_{\varphi} \equiv 0$ on $\left.P_{k}\right)$ and is $\mathbb{S}^{1} \times G$-invariant.

Recall that a function $f: M \rightarrow \mathbb{R}$ is called Morse-Bott if $\operatorname{Crit}(f)$ is a submanifold of $M$ and $T_{x} \operatorname{Crit}(f)=\operatorname{ker} \operatorname{Hess}_{x}(f)$ for all $x \in \operatorname{Crit}(f)$, where $\operatorname{Hess}_{x}(f): T_{x} M \rightarrow T_{x} M$ denotes the Hessian of $f$ at the point $x$.

In the case we are considering $\operatorname{Crit}\left(\mu_{\varphi}\right)=P_{k}$ : in fact, if $v \in P_{k}, X_{\varphi}(v)=0$, and hence $d \mu_{\varphi}=i_{X_{\varphi}} \omega=0$. Conversely, since $X_{\varphi}$ always lies in the fibre of $\nu_{k}$ and $\omega$ restricts to a symplectic form there, $d \mu_{\varphi}=i_{X_{\varphi}} \omega$ can only vanish if $X_{\varphi}=0$. It is easy to show that if $v \in P_{k}$, the inclusion $T_{v} \operatorname{Crit}\left(\mu_{\varphi}\right) \leq \operatorname{ker} \operatorname{Hess}_{v}\left(\mu_{\varphi}\right)$ holds.

To see that equality holds one needs to show that $\operatorname{dim} \operatorname{ker} \operatorname{Hess}_{v}\left(\mu_{\varphi}\right) \leq \operatorname{dim} P_{k}$ or, equivalently, that $\operatorname{rank} \operatorname{Hess}_{v} \mu_{\varphi}$ is at least equal to the rank of $\nu_{k}$. Restricting $\omega, \varphi$ and $\mu_{\varphi}$ to one fibre of $\nu_{k}$ we are in a proper symplectic situation and we may conclude that $\left.\mu_{\varphi}\right|_{\nu_{k}(x)}$ is Morse (see [MS98, Section 5.5]), hence in particular it has full rank. Introducing bundle coordinates on $U$ and computing the matrix of second derivatives of $\mu_{\varphi}$, which represent the Hessian in these coordinates, we see that it always contains a non singular block, corresponding to the above restriction of $\mu_{\varphi}$ to one fibre, having rank equal to the rank of $\nu_{k}$. This proves that $\mu_{\varphi}$ is Morse-Bott, and it only remains to show that it is positive outside $P_{k}$.

Let $\partial_{r}$ be the radial vector field on $\nu_{k}$ given by

$$
\partial_{r}(v)=\left.\frac{d}{d t}\right|_{t=1} t \cdot v
$$

for $v \in \nu_{k}$. We will show that $\mu_{\varphi}$ strictly increases in radial direction, more precisely that $\mathcal{L}_{\partial_{r}} \mu_{\varphi} \geq 0$ in $U$ (possibly after shrinking $U$ ) with equality only at the zero section. By definition of $\mu_{\varphi}$, one has $i_{\partial_{r}} d \mu_{\varphi}=\omega\left(X_{\varphi}, \partial_{r}\right)$, so it will suffice to show that there exists a neighbourhood of $P_{k}$ where $\omega\left(X_{\varphi}, \partial_{r}\right) \geq 0$. With $\pi$ denoting the bundle projection $\nu_{k} \rightarrow P_{k}$, the vertical bundle $V\left(\nu_{k}\right)$ of $\nu_{k}$ can be identified with the pull-back

$$
\pi^{*} \nu_{k}=\left\{(v, w) \in \nu_{k} \times \nu_{k} \mid \pi(v)=\pi(w)\right\} .
$$

The identification of $\pi^{*}\left(\nu_{k}\right)$ and $V\left(\nu_{k}\right)$ goes as follows

$$
\pi^{*}\left(\nu_{k}\right) \rightarrow V\left(\nu_{k}\right),\left.\quad(v, w) \mapsto \frac{d}{d t}\right|_{t=0}(v+t w) .
$$

Let $v \in \nu_{k}$, and write it as $v=v_{1}+\cdots+v_{l}$ with respect to the splitting $\nu_{k}=E_{1} \oplus \cdots \oplus E_{l}$. The vectors $X_{\varphi}$ and $\partial_{r}$ lie in $V\left(\nu_{k}\right) \cong \pi^{*}\left(\nu_{k}\right)$ and they are given by

$$
X_{\varphi}(v)=\left(v, a_{1} J v_{1}+\cdots+a_{l} J v_{l}\right) \quad \text { and } \quad \partial_{r}(v)=(v, v) .
$$

Let $w$ be another vector in $\nu_{k}$ with $\pi(v)=\pi(w),\|w\|=1$, and $w=w_{1}+\cdots+w_{l}$ with respect to the splitting $\nu_{k}=E_{1} \oplus \cdots \oplus E_{l}$. We will show that if $v$ lies in the zero section of $\nu_{k}$, then one has

$$
\omega\left(\left(v, \sum_{j=1}^{l} a_{j} J w_{j}\right),(v, w)\right)>0 .
$$

Then by continuity there exists a neighbourhood of the zero section of $\nu_{k}$ where this holds for all $\|w\|=1$ and therefore, by scaling, for all $w \neq 0$. Hence in particular $\omega\left(X_{\varphi}, \partial_{r}\right) \geq 0$ on this neighbourhood, with equality only at the zero section. In order to prove (3.2), recall that at the 
zero section of $\nu_{k}$ the differential of exp is the identity and the presymplectic form $\omega_{P}$ is left unchanged by averaging. So it follows that

$$
\omega\left(\left(v, \sum_{j=1}^{l} a_{j} J w_{j}\right),(v, w)\right)=\omega\left(\sum_{j=1}^{l} a_{j} J w_{j}, w\right)=\sum_{j=1}^{l} a_{j} \omega\left(J w_{j}, w_{j}\right)>0,
$$

since the eigenspaces $E_{j}$ 's are $\omega$-orthogonal.

3.3. Surgery along the minimal stratum. As in the previous section, consider the minimal singular stratum $P_{k}$, denote by $\nu_{k}$ its normal bundle in $P$ and take now the product $\nu_{k} \times \mathbb{C}$. We can extend the original $\beta$ - and $G$-action to this product by letting $\mathbb{S}^{1}$ and $G$ act trivially on the $\mathbb{C}$-factor, namely, for $v \in \nu_{k}(x), w \in \mathbb{C}$

$$
\lambda *_{\beta}(v, w):=\left(\lambda *_{\beta} v, w\right) \quad \text { and } \quad g *_{G}(v, w):=\left(g *_{G} v, w\right) .
$$

We can define a second circle action on $\nu_{k} \times \mathbb{C}$ by setting

$$
\lambda *_{\varphi}(v, w)=\left(\lambda *_{\varphi} v, \lambda^{-k} w\right)=\left(\left(\lambda^{a_{1}} v_{1}+\cdots+\lambda^{a_{l}} v_{l}\right), \lambda^{-k} w\right),
$$

where $v=v_{1}+\cdots+v_{l}$ is the splitting with respect to the eigenspaces defined above. The $\beta$ - and $\varphi$-actions commute and therefore we can combine them and define a new $\mathbb{S}^{1}$-action

$$
\lambda *_{\tau}(v, w):=\lambda *_{\varphi}\left(\lambda^{-1} *_{\beta}(v, w)\right) .
$$

This $\tau$-action is not effective, because the $\varphi$ - and the $\beta$-action coincide for elements in $\mathbb{Z}_{k}$. But consider the short exact sequence

$$
0 \rightarrow \mathbb{Z}_{k} \rightarrow \mathbb{S}^{1} \rightarrow \hat{\mathbb{S}}^{1} \rightarrow 0
$$

with the homomorphism of the circle given by $\lambda \mapsto \lambda^{k}$, and let $\hat{\mathbb{S}}^{1}$ act on $\nu_{k} \times \mathbb{C}$ by $\sigma *_{\hat{\tau}}(v, w)=$ $\lambda *_{\tau}(v, w)$ for some $\lambda \in \mathbb{S}^{1}$ such that $\lambda^{k}=\sigma$. This new action, which we denote by $\hat{\tau}$, is not only effective but even free and the quotient $\left(\nu_{k} \times \mathbb{C}\right) / \hat{\tau}$ is a smooth manifold. Since $\varphi$ and $G$ commute with $\hat{\tau}$ and with each other, they descend to this quotient, inducing an $\mathbb{S}^{1} \times G^{-}$ action, that is again locally free outside the set $\left\{[v, 0] \mid v \in P_{k}\right\}$. To see this, assume there is $[v, w] \in\left(\nu_{k} \times \mathbb{C}\right) / \hat{\tau}$ and a sequence $\left\{\left(\lambda_{n}, g_{n}\right)\right\}$ in $\mathbb{S}^{1} \times G$, converging to the identity element $(1, e)$, such that $\left(\lambda_{n}, g_{n}\right) *_{\varphi \times G}[v, w]=[v, w]$ for all $n \in \mathbb{N}$. This means that for each $n$ there exists a unique element $\sigma_{n} \in \mathbb{S}^{1}$ such that $\sigma_{n} \rightarrow 1$ and

$$
\left(\lambda_{n}, g_{n}\right) *_{\varphi \times G}(v, w)=\sigma_{n} *_{\hat{\tau}}(v, w) .
$$

By definition of the $\hat{\tau}$-action, $\sigma_{n} *_{\hat{\tau}}(v, w)=\mu_{n} *_{\tau}(v, w)$ for some sequence $\left\{\mu_{n} \in \mathbb{S}^{1}\right\}$ such that $\mu_{n}^{k}=\sigma_{n}$ and $\mu_{n} \rightarrow 1$. Dropping the $w$-coordinate and projecting onto the zero section of $\nu_{k}$ we obtain $g_{n} *_{G} \pi(v)=\mu_{n} *_{\beta} \pi(v)$, that is, $\left(\mu_{n}^{-1}, g_{n}\right) \in \operatorname{Stab}_{\beta \times G}(\pi(v))$. Since the $\beta \times G$-action is locally free, it follows that $\mu_{n}=1$ and $g_{n}=e$ for all $n$ sufficiently large. We can now rewrite Equation (3.3) as $\lambda_{n} *_{\varphi}(v, w)=(v, w)$ and from this conclude that either $\lambda=1$ or $(v, w)$ has to lie in $P_{k} \times\{0\}$.

We define a 2 -form $\Omega=(\omega,-i d w \wedge d \bar{w})$ on $\nu_{k} \times \mathbb{C}$, which is invariant with respect to the $\hat{\tau}$-action. By construction, the infinitesimal generator of this action can be written as $X_{\hat{\tau}}=\frac{1}{k}\left(-X_{\beta}+X_{\varphi}\right)$. The "Hamiltonian" for the $\hat{\tau}$-action, given by

$$
H_{\hat{\tau}}(v, w)=\frac{1}{k} \mu_{\varphi}(v)-|w|^{2},
$$

satisfies $i_{X_{\hat{\tau}}} \Omega=d H_{\hat{\tau}}$. It follows that if we restrict to a regular level set of $H_{\hat{\tau}}$, say $H_{\hat{\tau}}^{-1}(\varepsilon / k)$, we have $i_{X_{\hat{\tau}}} \Omega=0$. In other words, on such a level set the generator of the $\hat{\tau}$-action is contained in the kernel of the 2 -form. In fact, this kernel contains the subspace spanned by the infinitesimal generators of the $G^{-}, \varphi^{-}$, and $\hat{\tau}$-actions. For an arbitrary skew-symmetric two-form $\omega$ on a vector space $V$ and a linear subspace $W \leq V$, one has

$$
\operatorname{dim} W+\operatorname{dim} W^{\omega}=\operatorname{dim} V+\operatorname{dim}(W \cap \operatorname{ker} \omega) .
$$

With $V=T\left(\nu_{k} \times \mathbb{C}\right)$ and $W=T H_{\hat{\tau}}^{-1}(\varepsilon / k)$, using that ker $\Omega$ is spanned by the generators of the $G$ - and $\varphi$-actions, this formula gives $\operatorname{dim} W^{\Omega}=\operatorname{dim} G+2$, which implies that on $H_{\hat{\tau}}^{-1}(\varepsilon / k)$ the kernel of $\left.\Omega\right|_{W}$ coincides with the subspace spanned by the infinitesimal generators of the $G^{-}, \varphi^{-}$, 
and $\hat{\tau}$-actions. Hence the quotient $P_{\varepsilon}:=H_{\varphi}^{-1}(\varepsilon / k) / \hat{\tau}$, with the structure induced by $\Omega, \varphi$, and $G$ is a smooth presymplectic $\mathbb{S}^{1} \times G$-manifold.

Notice that $H_{\hat{\tau}}^{-1}(\varepsilon / k)$ can be written as the disjoint union of two $\hat{\tau}$-invariant manifolds

$$
H_{\hat{\tau}}^{-1}(\varepsilon / k)=\left\{\left.(v, w)\left|\mu_{\varphi}(v)>\varepsilon,\right| w\right|^{2}=\frac{\mu_{\varphi}(v)-\varepsilon}{k}\right\} \sqcup\left\{(v, 0) \mid \mu_{\varphi}(v)=\varepsilon\right\} .
$$

Choose $\delta>0$ such that $\mu_{\varphi}^{-1}(\delta)$ is contained in $U$, the neighbourhood of $P_{k}$ constructed in Proposition 4 . Notice that $\mu_{\varphi}^{-1}(\delta)$ has the structure of a sphere bundle over $P_{k}$. For $0<\varepsilon<\delta$, denote by $\nu_{k}(\varepsilon)$ the subset of $\nu_{k}$ given by $\left\{\mu_{\varphi}(v)<\varepsilon\right\}$, by $\nu_{k}(\varepsilon, \delta)$ the "annulus" $\left\{v \in \nu_{k} \mid \varepsilon<\mu_{\varphi}(v)<\delta\right\}$, and consider the map

$$
\Phi: \nu_{k}(\varepsilon, \delta) \rightarrow P_{\varepsilon}, \quad v \mapsto\left[v, \sqrt{\frac{\mu_{\varphi}(v)-\varepsilon}{k}}\right] .
$$

This is an $\mathbb{S}^{1} \times G$-equivariant diffeomorphism (onto its image), where the $\mathbb{S}^{1}$-action is the $\beta$-action on $\nu_{k}$ and the $\varphi$-action on $P_{\varepsilon}$. Its inverse can be constructed as follows: given $[v, w]$ with $w \neq 0$, we first represent the same class by an element $\left(v^{\prime}, w^{\prime}\right)$ such that $w^{\prime}$ is a real positive number, and then define

$$
\Phi^{-1}([v, w]):=v^{\prime} .
$$

Moreover, since $\Phi$ factors through the map $\nu_{k}(\varepsilon, \delta) \hookrightarrow H_{\hat{\tau}}^{-1}(\varepsilon / k)$ which is the identity in the first component and a real function in the second one, we have

$$
\Phi^{*} \Omega=\Phi^{*}(\omega,-i d w \wedge d \bar{w})=\omega,
$$

hence $\Phi$ gives in fact an equivariant presymplectic identification of $\nu_{k}(\varepsilon, \delta)$ with its image under $\Phi$. More precisely we have

$$
\Phi\left(\nu_{k}(\varepsilon, \delta)\right)=\left\{[v, w] \in P_{\varepsilon} \mid \varepsilon<\mu_{\varphi}(v)<\delta\right\} .
$$

We can now remove a tubular $\varepsilon-$ neighbourhood of $P_{k}$ in $\nu_{k}$ and glue in the smooth manifold

$$
V(\delta):=\left\{\left.(v, w)\left|\varepsilon \leq \mu_{\varphi}(v)<\delta,\right| w\right|^{2}=\frac{\mu_{\varphi}(v)-\varepsilon}{k}\right\} / \hat{\tau}
$$

along the open "collar" $\nu_{k}(\varepsilon, \delta)$, using the map $\Phi$. In this way we define the new manifold

$$
\widetilde{P}=\left(P-\overline{\nu_{k}(\varepsilon)}\right) \bigcup_{\Phi} V(\delta) .
$$

Since $\Phi$ is equivariant, the $\beta$-action on $P-\overline{\nu_{k}(\varepsilon)}$ and the $\varphi$-action on $V(\delta)$ fit together to give a circle action $\tilde{\beta}$ on $\widetilde{P}$, which by construction coincides with $\beta$ outside a $\delta$-neighbourhood of $P_{k}$. Similarly, the $G$-actions can be combined to define a $G$-action on $\widetilde{P}$. Moreover, $\Phi$ identifies the given closed 2 -forms on the two sides of the gluing, so $\widetilde{P}$ also admits a closed 2 -form $\widetilde{\omega}$ with the property that $\widetilde{\omega}=\omega_{P}$ on $P-\nu_{k}(\delta)$. With the action of $\mathbb{S}^{1} \times G$ and the $2-$ form $\widetilde{\omega}$ just defined, $\widetilde{P}$ is a presymplectic manifold.

Moreover, there exists a map $f: \widetilde{P} / \tilde{\beta} \rightarrow P / \beta$, which is a $G$-equivariant presymplectic orbifold isomorphism outside an arbitrarily small neighbourhood of $P_{k}$ (and in fact coincides with the identity map outside a slightly larger neighbourhood). We shall describe how to define $f$. On $\left(P-\overline{\nu_{k}(\delta)}\right) / \beta$ it is simply the identity. In order to define it on $V(\delta) / \varphi$ a little more work is required. First of all, denote by $S_{\varepsilon}$ the quotient $\left\{(v, 0) \in H_{\hat{\tau}}^{-1}(\varepsilon) \mid \mu_{\varphi}(v)=\varepsilon\right\} / \hat{\tau}$. Then the inverse of the gluing map $\Phi$ gives us a diffeomorphism $\Phi^{-1}: V(\delta)-S_{\varepsilon} \rightarrow \nu_{k}(\varepsilon, \delta)$. Since $\Phi$ is equivariant with respect to the $\varphi \times G-$ and $\beta \times G$-actions, this descends to a $G$-equivariant presymplectic orbifold isomorphism $\left(V(\delta)-S_{\varepsilon}\right) / \varphi \rightarrow \nu(\varepsilon, \delta) / \beta$. Let $h:[0, \infty) \rightarrow[0, \infty)$ be a smooth monotone real function, which satisfies the following three conditions:

(i) $h(t)=0$ for all $t \leq \varepsilon$;

(ii) $h$ is strictly increasing on $(\varepsilon, \delta)$;

(iii) $h(t)=1$ for all $t \geq \delta$. 
For $v \in \nu_{k}(\varepsilon, \delta)$, define

$$
\widetilde{S}: \nu_{k}(\varepsilon, \delta) \rightarrow \nu_{k}(\delta)-P_{k}, \quad v \mapsto h\left(\mu_{\varphi}(v)\right) \cdot v
$$

The map $\widetilde{S}$ is $\beta \times G$-equivariant so it descends to a well-defined $G$-equivariant map

$$
S: \nu_{k}(\varepsilon, \delta) / \beta \rightarrow\left(\nu_{k}(\delta)-P_{k}\right) / \beta \text {. }
$$

The composition of $\Phi^{-1}$ with the "stretching" map $S$ yields a map

$$
f:\left(V(\delta)-S_{\varepsilon}\right) / \varphi \rightarrow\left(\nu_{k}(\delta)-P_{k}\right) / \beta .
$$

Because of the boundary conditions on $h$, the map $f$ glues on the outer side with the identity map on $\left(P-\nu_{k}(\delta)\right) / \beta$. On the inner side we extend it as the map $S_{\varepsilon} / \varphi \rightarrow P_{k} / \beta$, which sends the $\varphi-$ orbit of $[v, 0]$ to the $\beta$-orbit of $\pi(v)$ ( $\pi$ being the projection of $\nu_{k}$ to its zero section). To see that $f$ is continuous in a neighbourhood of $S_{\varepsilon} / \varphi$, one has to show that for any sequence $\left[v_{k}, w_{k}\right] / \varphi \subset V(\delta) / \varphi$ that converges to some element $[v, 0] / \varphi$, it follows that $f\left(\left[v_{k}, w_{k}\right] / \varphi\right)$ converges to $f([v, 0] / \varphi)$. We can find representatives $\left(v_{k}^{\prime}, w_{k}^{\prime}\right) \in H_{\hat{\tau}}^{-1}(\varepsilon / k)$ for the sequence that converge to $\left(v^{\prime}, 0\right)$, and hence $f\left(\left[v_{k}^{\prime}, w_{k}^{\prime}\right] / \varphi\right)=\left(h\left(\mu_{\varphi}\left(v_{k}^{\prime}\right)\right) \cdot v_{k}^{\prime}\right) / \beta$ converges to $\pi\left(v^{\prime}\right) / \beta=\pi(v) / \beta$.

\section{Resolution of Symplectic Cuts}

The methods described above can also be applied in some situations to find resolutions of symplectic cuts, thus giving a (positive) answer to a question posed by River Chiang and Eugene Lerman.

First note that the construction in the proof of Lemma 2 can also be carried out so as to preserve an additional $K$-action. If $(P, \omega)$ is a presymplectic $\mathbb{S}^{1} \times G$-manifold as in Lemma 2 with an additional $K$-action that preserves $\omega$ and commutes with $\mathbb{S}^{1} \times G$, then for an arbitrarily small neighborhood $U$ of the singular set of the circle action we find a presymplectic $\mathbb{S}^{1} \times G^{-}$ manifold $(\widetilde{P}, \widetilde{\omega})$ with a $K$-action such that the $\mathbb{S}^{1}$-action is free, and such that there is a map $p: \widetilde{P} / \mathbb{S}^{1} \rightarrow P / \mathbb{S}^{1}$ that is a $G$-equivariant presymplectic resolution of $P / \mathbb{S}^{1}$ supported on $U$. The projection $p$ is also $K$-equivariant, and if the initial $K$-action was "Hamiltonian" with a moment map $\mu_{K}$ such that $\iota_{Y_{M}} \omega=d\left\langle\mu_{K} \mid Y\right\rangle$ for every $Y \in \mathfrak{k}$, then we will also recover a moment map $\widetilde{\mu}_{K}$ on the resolution that coincides with $\mu_{K}$ outside the neighborhood $U$.

To simplify the presentation in the proof, we chose not to include such a $K$-action: With this additional ingredient, most steps of the proof just go through in a straightforward way, but let us remark that in Proposition 4 , the function $\mu_{\varphi}$ is $K$-invariant, because

$$
d\left(\mathcal{L}_{Y_{P}} \mu_{\varphi}\right)=\mathcal{L}_{Y_{P}}\left(\iota_{X_{\varphi}} \omega\right)=0
$$

for every infinitesimal generator $Y_{P}$ associated to $Y \in \mathfrak{k}$. It follows that $\mathcal{L}_{Y_{P}} \mu_{\varphi}=$ const, and since $Y_{P}$ is tangent to the stratum $P_{k}$, and $\mu_{\varphi}$ vanishes on $P_{k}$, we have $\mathcal{L}_{Y_{P}} \mu_{\varphi}=0$. For the proof of Proposition 3 in the appendix, average $\alpha$ also over the group $K$, and show that $X_{s}$ commutes with the generators of $K$ in the same way as it was done for the $G$-action. The moment map $\widetilde{\mu}_{K}$ on the patch $V(\delta)$ is given by projection onto the first factor, followed by the original moment map $\mu_{K}$.

Theorem 5. Let $(M, \omega)$ be a Hamiltonian $\mathbb{S}^{1}$-manifold with Hamiltonian function $h: M \rightarrow \mathbb{R}$. Assume that $a \in h(M)$ is a regular value of $h$. Then we can perform the symplectic cut at a to obtain the symplectic orbifolds

$$
M_{(-\infty, a]} \quad \text { and } \quad M_{[a, \infty)} .
$$

For any $\varepsilon>0$, there are symplectic resolutions of both orbifolds by symplectic $\mathbb{S}^{1}$-manifolds $\left(\widetilde{M}_{-}, \omega_{-}\right)$and $\left(\widetilde{M}_{+}, \omega_{+}\right)$with Hamiltonians $\widetilde{H}_{-}$and $\widetilde{H}_{+}$, respectively, such that $h^{-1}(-\infty, a-\varepsilon)$ is isomorphic to $\widetilde{H}_{-}^{-1}(-\infty, a-\varepsilon)$ and $h^{-1}(a+\varepsilon, \infty)$ to $\widetilde{H}_{+}^{-1}(a+\varepsilon, \infty)$.

Proof. The symplectic cut is performed by taking the product manifold $M \times \mathbb{C}$ with symplectic form $\omega \oplus-i d z \wedge d \bar{z}$ and circle action $e^{i \varphi} *(p, z):=\left(e^{i \varphi} p, e^{i \varphi} z\right)$ or $e^{i \varphi} *(p, z):=\left(e^{i \varphi} p, e^{-i \varphi} z\right)$, depending on whether one wants to produce $M_{(-\infty, a]}$ or $M_{[a, \infty)}$. In the first case, the Hamiltonian 
function will be $H_{-}(p, z)=H(p)+|z|^{2}$, in the second it will be $H_{+}(p, z)=H(p)-|z|^{2}$. Taking the symplectic reduction at the value $a$ will yield the symplectic cut spaces.

Let us now focus on the 'positive' part of the cut, $M_{[a, \infty)}$, since the argument for the negative part is completely analogous. There is a symplectic embedding of $h^{-1}(a+\varepsilon, \infty)$ into $M_{[a, \infty)}$, defined by $p \mapsto[p, \sqrt{h(p)-a}]$ : it is equivariant with respect to the circle action induced on $M_{[a, \infty)}$ by $e^{i \varphi} *(p, z):=\left(e^{i \varphi} p, z\right)$. Notice that this action on the presymplectic manifold $H_{+}^{-1}(a)$ plays the role of the additional $K$-action mentioned above.

The potential orbifold singularities of $M_{[a, \infty)}$ lie in the cut hypersurface $\left\{[p, 0] \mid p \in h^{-1}(a)\right\}$. Let $U_{\varepsilon}$ be the neighbourhood of this set defined as the complement of the image of $\{(p, z) \mid h(p) \geq$ $a+\varepsilon,|z|=\sqrt{h(p)-a}\}$. Denote by $\widetilde{M}_{+}$the resolution of $M_{[a, \infty)}$ supported on $U_{\varepsilon}$ obtained with the method of the previous section and preserving the additional circle action (the $K$-action). Then $h^{-1}(a+\varepsilon, \infty)$ embeds into $\widetilde{M}_{+}$in a symplectic and equivariant way. By construction, the Hamiltonian for the $K$-action on $\widetilde{M}_{+}$coincides with $h$ outside the neighbourhood $U_{\varepsilon}$, so the image of this embedding coincides with $\widetilde{H}_{+}^{-1}(a+\varepsilon, \infty)$.

\section{Appendix A. Proof of Proposition 3}

The restrictions of $\omega$ and $\omega_{P}$ coincide along the zero section of $\nu_{k}$. In particular, if we denote by $i_{0}: P_{k} \hookrightarrow \nu_{k}$ the inclusion of $P_{k}$ as the zero section of $\nu_{k}$, we have

$$
i_{0}^{*}\left(\omega-\omega_{P}\right)=0 .
$$

This implies that there exists a 1 -form $\alpha$ on $\nu_{k}$ such that $\omega-\omega_{P}=d \alpha$ and moreover $\alpha$ vanishes on $T_{x} \nu_{k}$ for every $x \in P_{k}$ (see for example CdS01, Theorem 6.8] or [MS98, Lemma 3.14]). Furthermore, we may assume $\alpha$ to be $\beta$ - and $G$-invariant, because if it were not, we could replace it by its average

$$
\alpha^{\prime}=\int_{\mathbb{S}^{1} \times G}\left(g^{*} \lambda^{*} \alpha\right) d \lambda d g
$$

which still satisfies

$$
d \alpha^{\prime}=\int_{\mathbb{S}^{1} \times G}\left(g^{*} \lambda^{*} d \alpha\right) d \lambda d g=\int_{\mathbb{S}^{1} \times G} g^{*} \lambda^{*}\left(\omega-\omega_{P}\right) d \lambda d g=\omega-\omega_{P}
$$

and $\alpha^{\prime}=0$ on points of $P_{k}$. Let $Y$ be an arbitrary element in the Lie algebra of $\mathbb{S}^{1} \times G$, and let $Y_{P}$ be its infinitesimal generator. Notice that from the $\mathbb{S}^{1} \times G$-invariance, we also obtain that $i_{Y_{P}} \alpha=$ const, but since $i_{Y_{P}} \alpha=0$ on the zero section, it follows that $Y_{P}$ lies everywhere in the kernel of $\alpha$.

Now define the 1 -parameter family of presymplectic 2 -forms

$$
\omega_{s}:=s \omega+(1-s) \omega_{P} .
$$

Assume there exists a time-dependent vector field $X_{s}$ such that $\omega=\left(\Phi_{s}^{X_{s}}\right)^{*} \omega_{s}$, where $\Phi_{s}^{X_{s}}$ denotes the flow of $X_{s}$. Then we have

$$
0=\frac{d}{d s}\left(\Phi_{s}^{X_{s}}\right)^{*} \omega_{s}=\left(\Phi_{s}^{X_{s}}\right)^{*}\left(\mathcal{L}_{X_{s}} \omega_{s}+\frac{d}{d s} \omega_{s}\right)
$$

which is equivalent to $\mathcal{L}_{X_{s}} \omega_{s}+d \alpha=0$, or

$$
d\left(i_{X_{s}} \omega_{s}+\alpha\right)=0 .
$$

In order to show that $\omega$ and $\omega_{P}$ are $\mathbb{S}^{1} \times G$-equivariantly isomorphic, we need to find $X_{s}$ satisfying the last equation, integrating to a time- 1 flow in a neighbourhood of $P_{k}$ and commuting with $\mathbb{S}^{1} \times G$.

Let $X_{s}$ be the unique vector field determined by

$$
\left\{\begin{array}{l}
g\left(Y_{P}, X_{s}\right)=0 \Leftrightarrow X_{s} \in \Omega^{P}=\left(\operatorname{ker} \omega_{P}\right)^{\perp} \\
\left.\left(i_{X_{s}} \omega_{s}+\alpha\right)\right|_{\Omega^{P}}=0
\end{array}\right.
$$


for any infinitesimal generator $Y_{P}$ for the $\mathbb{S}^{1} \times G$-action. It is easy to see that $i_{Y_{P}}\left(i_{X_{s}} \omega_{s}+\alpha\right)=0$, so it follows that $i_{X_{s}} \omega_{s}+\alpha=0$ on the whole tangent space and not just on $\Omega^{P}$. To see that $X_{s}$ commutes with $X_{\beta}$, compute

$$
0=\mathcal{L}_{Y_{P}}\left(i_{X_{s}} \omega_{s}+\alpha\right)=i_{\mathcal{L}_{Y_{P}} X_{s}} \omega_{s}
$$

and

$$
0=\mathcal{L}_{Y_{P}}\left(g\left(X_{s}, Y_{P}\right)\right)=g\left(Y_{P}, \mathcal{L}_{Y_{P}} X_{s}\right) .
$$

Combining this with the fact that $\omega_{s}$ is nondegenerate on $\Omega^{P}$ we get that $\mathcal{L}_{Y_{P}} X_{s}=0$.

Finally notice that, since $\alpha$ vanishes at all points of the zero section of $\nu_{k}, X_{s}$ also does and hence its flow is defined in a neighbourhood of this section up to time one.

\section{REFERENCES}

[CdS01] A. Cannas da Silva, Lectures on symplectic geometry., Lecture Notes in Mathematics. 1764. Berlin: Springer. xii, 217 p., 2001.

[Ler95] E. Lerman, Symplectic cuts, Math. Res. Lett. 2 (1995), no. 3, 247-258.

[LT97] E. Lerman and S. Tolman, Hamiltonian torus actions on symplectic orbifolds and toric varieties, Trans. Amer. Math. Soc. 349 (1997), no. 10, 4201-4230. MR MR1401525 (98a:57043)

[MS98] D. McDuff and D. Salamon, Introduction to symplectic topology. 2nd ed., Oxford Mathematical Monographs. New York, NY: Oxford University Press., 1998.

[NP07] K. Niederkrüger and F. Pasquotto, Resolution of symplectic cyclic orbifold singularities, arXiv math/0707.4141 (2007).

E-mail address, K. Niederkrüger: kniederk@umpa.ens-lyon.fr

E-mail address, F. Pasquotto: pasquott@few.vu.nl

(K. Niederkrüger) École Normale Supérieure de Lyon, Unité de Mathématiques Pures et Appliquées, UMR CNRS 5669, FRANCE

(F. Pasquotto) Department of Mathematics, Faculty of Sciences, Vrije Universiteit, De Boelelaan 1081A, 1081 HV Amsterdam, The Netherlands 